\title{
Identification of OSBPL2 as a novel candidate gene for progressive nonsyndromic hearing loss by whole-exome sequencing
}

\author{
Guangqian Xing, MD1, Jun Yao, PhD², Bin Wu, MSc33 Tingting Liu, MD'1, Qinjun Wei, MD², Cheng Liu, MD', \\ Yajie Lu, MSc', Zhibin Chen, MD', Heng Zheng, $\mathrm{PhD}^{4}$, Xiaonan Yang, $\mathrm{MSc}^{3}$ and Xin Cao, $\mathrm{PhD}^{2}$
}

\begin{abstract}
Purpose: Various forms of hearing loss have genetic causes, but many of the responsible genes have not yet been identified. Here, we describe a large seven-generation Chinese family with autosomal dominant nonsyndromic hearing loss that has been excluded as being caused by known deafness gene mutations associated with autosomal dominant nonsyndromic hearing loss with the aim of identifying a novel causative gene involved in deafness.
\end{abstract}

Methods: Whole-exome sequencing was conducted in three affected family members, and cosegregation analysis was performed on other members of the family.

Results: Whole-exome sequencing and subsequent segregation analysis identified a heterozygous frameshift mutation (c.153_154delCT, p.Gln53Argfs $\left.{ }^{\star} 100\right)$ in the oxysterol binding protein-like 2 (OSBPL2) gene in 25 affected family members. The deletion mutation is predicted to lead to premature truncation of the OSBPL2 protein. Modeling and structure-based analysis support the theory that this gene deletion is functionally deleterious. Our finding was further confirmed by the detection of another missense mutation, a c.583C $>$ A transversion (p.Leu195Met) in exon 7 of OSBPL2, in an additional sporadic case of deafness.

Conclusion: Based on this study, OSBPL2 was identified as an excellent novel candidate gene for autosomal dominant nonsyndromic hearing loss; this study is the first to implicate OSBPL2 mutations in autosomal dominant nonsyndromic hearing loss.

Genet Med advance online publication 31 July 2014

Key Words: autosomal dominant inheritance; nonsyndromic hearing loss; OSBPL2; whole-exome sequencing

\section{INTRODUCTION}

Hearing loss is one of the most common sensorial disorders, with an incidence of $\sim 1$ in 1,000 children. It is estimated that at least half of the cases are attributable to genetic factors, and more than two-thirds of this subset of cases are classified as nonsyndromic hearing loss (NSHL) because of the absence of additional symptoms. ${ }^{1}$ NSHL is reportedly associated with different genes in autosomal dominant (AD), autosomal recessive, $\mathrm{X}$-linked, and maternal inheritance patterns. To date, more than 64 genes have been linked to NSHL, including more than $27 \mathrm{AD}$ NSHL genes. Because of the striking genetic heterogeneity of hearing loss and the exquisite sensitivity of the human auditory system, however, it is estimated that the genetic causes listed above explain only less than half of the clinical cases in inheritance patterns $\mathrm{s}^{2,3}$ (http://hereditaryhearingloss.org/). Therefore, many patients and families with hereditary hearing loss have not received an explanation for additional unknown causative genes.

Whole-exome sequencing (WES) has been successfully used to find disease-causing mutations in Mendelian disorders. ${ }^{4-19}$ It is estimated that more than 230 novel rare disease genes have been discovered to date using WES. ${ }^{11}$ Several new NSHLcausative genes have also recently been revealed via WES, including GPSM2, DNMT1, BDP1, ELMOD3, TNC, GRXCR2, and $A D C Y 1{ }^{12-18}$ Thus, WES is a powerful approach for investigating the genetic basis of human disease.

In the current study, using WES we identified a novel candidate AD NSHL-causative gene, oxysterol binding proteinlike 2 (OSBPL2; NC_000020.11, NM_144498.2, NP_653081.1, OMIM: 606731), in a large Chinese family (JSNY-028). Four relatives (three affected individuals and one healthy spouse) of this family were included in the WES. Several lines of evidence supported the candidate role of this gene, including (i) a heterozygous frameshift mutation (c.153_154delCT) in exon 3 of OSBPL2 was present in 25 available affected cases and absent in 26 unaffected family members, which completely cosegregated with the clinical phenotype of hearing loss in this family; (ii) OSBPL2 mutations were not detected in 300 unaffected individuals of matched ethnicity and geographic ancestry; (iii) a missense mutation (c.583C $>\mathrm{A}$ ) in exon 7 of OSBPL2 was detected in one of the 452 sporadic NSHL cases; (iv) the modeled structure of OSBPL2 also predicted that these two mutations were

The first three authors contributed equally to this work.

${ }^{1}$ Department of Otolaryngology, The First Affiliated Hospital of Nanjing Medical University, Nanjing, P.R. China; ${ }^{2}$ Department of Biotechnology, School of Basic Medical Science, Nanjing Medical University, Nanjing, P.R. China; ${ }^{3}$ BGI-Shenzhen, Guangdong, P.R. China; ${ }^{4}$ Department of Bioinformatics, School of Life Science and Technology, China Pharmaceutical University, Nanjing, P.R. China. Correspondence: Xin Cao (caoxin@njmu.edu.cn) 
functionally deleterious; and (v) strong expression of Osbpl2 was detected in mouse cochlea. To our knowledge, this is the first report that shows that OSBPL2 is a candidate causative gene for pedigrees and sporadic cases with NSHL and that it is also the first AD NSHL-candidate gene identified on human chromosome 20.

\section{MATERIALS AND METHODS Subjects and clinical investigation}

A large, seven-generation Chinese family of Han origin (designated as JSNY-028) with AD NSHL was recruited by a clinical follow-up in August 2010, and the clinical data of all affected members were updated (Supplementary Table S1 online; Supplementary Figure S1 online). A pedigree of the family is shown in Figure 1. Of note, 199 members in seven generations (176 members in four living generations) were described, and 45 living members of the family were diagnosed as having hereditary, progressive, sensorineural hearing loss without additional clinical symptoms. In this study blood samples from 25 affected individuals and 26 unaffected family members were collected and available for molecular analysis. All of the affected subjects underwent complete clinical examination and audiological evaluations, including pure-tone audiometry, immittance, auditory brainstem response, and distortion production otoacoustic emissions. The audiological data were evaluated based on the criteria established by the European Working Group on Genetics of Hearing Loss. A detailed medical history was also collected, including degree of hearing loss, age at onset, evolution of hearing impairment, use of hearing aids, presence of tinnitus, medication, noise exposure, pathologic changes in the ear, and other relevant clinical manifestations.

Thirty-eight probands with hereditary hearing loss and 452 sporadic patients were recruited for future analysis. All 38 selected families exhibited typical AD NSHL, and audiological assessments of the affected individuals revealed bilateral, progressive, sensorineural hearing loss. A total of 452 sporadic cases with NSHL participated in this study, including 250 deaf individuals from the Nanjing City School for Deaf Children and 202 cases from the otology clinic of the First Affiliated Hospital of Nanjing Medical University. The ages of the sporadic subjects varied from 2 to 42 years old. Family history and clinical questionnaires were obtained from all subjects or their parents. The hearing level of all of the participants was evaluated by audiological testing, including pure-tone audiometry and auditory brainstem response. The recruited subjects matched the following criteria: (i) having nonsyndromic sensorineural hearing loss, with the severity of hearing loss ranging from mild to profound; (ii) having no detectable etiology according to medical records; (iii) GJB2, SLC26A4, and mitochondrial DNA A1555G mutations, which are prevalent in the Chinese hearing loss population, were excluded by standard genetic screening; and (iv) all subjects or their parents gave written, informed consent. The analysis also included 300 normalhearing individuals as a matched control. All subjects recruited for the current study were of nonrelated geographic ancestry and of Han Chinese ethnicity.
This study was approved by the ethics committee of Nanjing Medical University, Jiangsu Province, and written consent was obtained from all participants or their guardians. All procedures used in this study conformed to the tenets of the Declaration of Helsinki.

Whole-blood samples, including those from 51 members of the large family ( 25 available affected individuals, 14 unaffected relatives, and 12 spouses), 38 probands from unrelated $\mathrm{AD}$ NSHL pedigrees, 452 sporadic NSHL cases, and 300 ethnicitymatched controls, were obtained. All DNA samples used for the study were extracted from whole blood.

To identify the deafness-causative mutation in JSNY-028, the reported causal genes of AD NSHL (http://hereditaryhearingloss.org/; accessed: June 2011) were included in mutation screening by polymerase chain reaction (PCR)-based direct sequencing. Considering that no variants of these genes were detected in two affected individuals (V:5, V: 20), it was suggested that an unknown mutation was responsible for AD NSHL.

\section{Exome sequencing analysis}

Three affected individuals (V: 5, VI: 4, and VI: 32) and one normal-hearing family member (V: 4) were included in the WES study. The genomic DNA extracted from whole blood was fragmented, and the exomes were captured using the Agilent SureSelect Human All Exon Kit (Agilent, Santa Clara, CA). The captured DNA was sequenced with 90-base pair (bp) pairedend reads on an Illumina HiSeq 2000 sequencing platform (Illumina, San Diego, CA) according to the manufacturer's protocol, and it was ensured that each sample was covered to an average sequencing depth of at least 80 -fold. Raw image files were processed by Illumina base-calling software version 1.7 for base calling with default parameters. SOAPaligner was used to align the sequenced raw data to the UCSC hg19 Burrows-Wheeler Aligner (0.5.9), and SOAPsnp was used to calculate the likelihood of possible genotypes in target regions. The low-quality variations were filtered out using the following criteria: (i) a quality score of $<20$; (ii) an average copy number at the allele site $\geq 2$; (iii) a distance between two adjacent single-nucleotide polymorphisms (SNPs) $<5 \mathrm{bp}$; and (iv) a sequencing depth $<4$ or $>500$. Insertions and deletions (indels) in the exome regions were aligned through the raw reads, and the alignment results were passed to the Genome Analysis Toolkit (Unified Genotyper; version 1.0) to identify the breakpoints. Indels were annotated with SeattleSeq Annotation 134 (http://snp.gs.washington.edu/SeattleSeqAnnotation134/) with respect to the relative position of a gene and their effects.

All single-nucleotide variants and indels were subsequently filtered against exome data from the dbSNP135 (http://www. genome.ucsc.edu/cgi-bin/hgGateway); the 1000 Genomes Project (http://www.1000genomes.org/); the HapMap database (http://www.hapmap.org, HapMap Data Release 24/phase II on the NCBI B36 assembly), with a minor allele frequency of $>0.5 \%$; and the YH database (http://yh.genomics.org.cn). SIFT (http://sift.jcvi.org) was used to predict whether an amino acid substitution would affect protein function. 


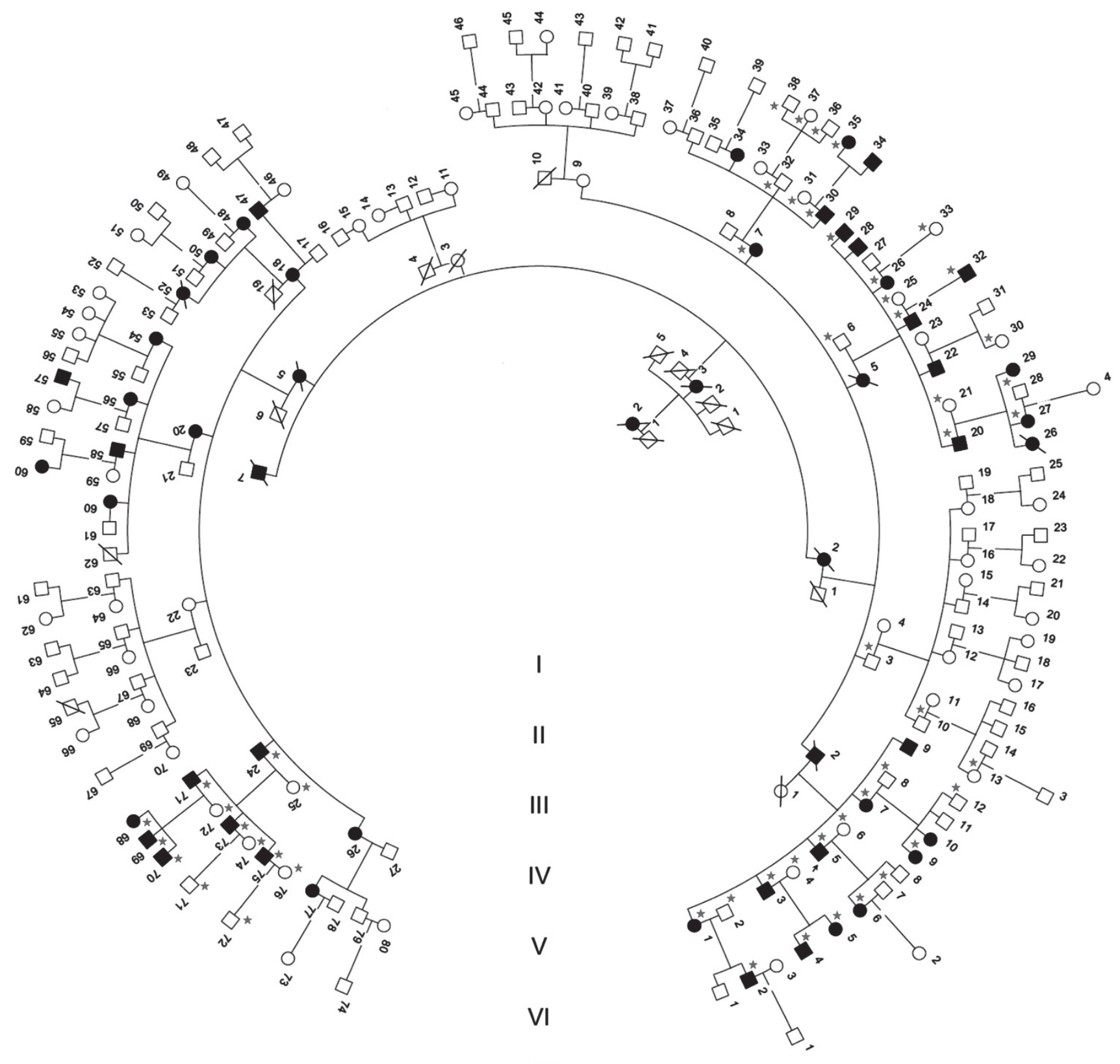

VII

Figure 1 Pedigree of JSNY-028. Filled symbols for males (squares) and females (circles) represent affected individuals; empty symbols represent unaffected ones. An arrow denotes the proband (V: 5). Symbols (pentagrams) indicate the available individuals for this study.

\section{Confirmation of OSBPL2 mutation}

Sanger sequencing was used to determine whether any of the candidate gene variants cosegregated with the disease phenotype in this family. Primers flanking the candidate loci were designed based on genomic sequences of the Human Genome and synthesized by BGI-Beijing, Shenzhen, China. All shared variants of the three affected individuals after filtering were then confirmed by direct PCR and sequencing (ABI 3730; Applied Biosystems, Foster City, CA). Sequencing data were compared pairwise with the Human Genome database. The primers flanking all exons and flanking introns of OSBPL2 were designed using Primer Premier 5.0 software (Premier, Palo Alto, CA) (Supplementary Table S2 online). Exon 3 and all other exons of OSBPL2, including exon-intron boundaries (derived from 51 members of the family JSNY-028, 38 probands from unrelated AD NSHL pedigrees, and 452 sporadic cases), were analyzed using the same method as described above. The sequences obtained were identified by sequence alignment with the NCBI Reference Sequence, and nucleotide changes were numbered corresponding to their position in OSBPL2 messenger RNA (NM_144498.2). 


\section{Three-dimensional structure modeling of OSBPL2}

The amino acid identity between OSBPL2 and oxysterol binding protein homolog 3 (Osh3) in this region was 36\%. Therefore, the holo-form of Osh3 binding with phosphatidylinositol 4-phosphate (PI[4]P) was used as a template (Protein Data Bank ID: 4INQ), and homology modeling was used to model the structure ofOSBPL2. The three-dimensional structure of OSBPL2 was modeled using MOE 2009 (Chemical Computing Group, Montreal, Quebec, Canada). The force field was set to CHARMM27, and $\mathrm{C}$-terminal and $\mathrm{N}$-terminal outgap modeling was carried out. The resulting structure was evaluated using PROCHECK and ERRAT (http://nihserver.mbi.ucla.edu/SAVES3/).

\section{Osbpl2 messenger RNA analysis in a mouse}

The cochlea, as well as the heart, liver, spleen, lung, kidney, muscle, and brain, were removed from a 12 -week-old mouse (C57BL/6), and total RNA was extracted with Trizol (Invitrogen, Carlsbad, CA). Reverse transcription was performed using the manufacturer's protocol (Invitrogen), and complementary DNA was synthesized according to standard conditions. The primers used for Osbpl2 (NM_144500.3) and $\beta$-actin (as the reference control) were designed using Primer Premier 5.0 software (Supplementary Table S3 online). The reverse transcription polymerase chain reactionPCR products were confirmed by direct sequencing.

\section{Tissue preparation and immunohistochemistry}

The separated mouse $(\mathrm{C} 57 \mathrm{BL} / 6)$ cochlea was fixed in $4 \%$ paraformaldehyde overnight, washed with phosphate-buffered saline, decalcified with $14 \%$ ethylenediaminetetraacetic acid ( $\mathrm{pH}$ 7.2) for 7 days, transferred to a series of gradient ethanol solutions, then immersed in dimethylbenzene and finally embedded in paraffin for sectioning. For immunohistochemical studies, the sections were deparaffinized in 3, 3 -diaminobenzidine and rehydrated in gradient ethanol solutions. Slides were then incubated overnight with rabbit antiOsbpl2 antibody (14751-1-AP; Proteintech, Chicago, IL) at a dilution of 1:200 in blocking buffer (a control without primary antibody was used). After rinsing, the slides were incubated for $1 \mathrm{~h}$ with horseradish peroxidase-conjugated goat anti-rabbit
IgG (SC-2004; Santa Cruz Biotechnology, Santa Cruz, CA) at a dilution of 1:100 in blocking buffer. The slides were washed with phosphate-buffered saline, stained with 3,3'-diaminobenzidine, and finally observed under a Leica upright microscope (DM 4000B; Leica Microsystems, Wetzlar, Germany).

\section{RESULTS}

\section{Family recruitment and clinical assessment}

The pedigree of family JSNY-028 spans seven generations and shows AD inheritance with no evidence of incomplete penetrance. Fifty-one available family members, including 25 affected members and 26 healthy members, participated in this study. The clinical features of the 25 affected family members are summarized in Supplementary Table $\mathbf{S 1}$ online. The age at onset of hearing impairment in these patients varied from 5 to 32 years. Pure-tone audiograms showed that all patients exhibited bilaterally symmetric, mild to profound sensorineural hearing loss. The hearing loss usually started at high frequencies at an early age and progressed rapidly to other frequencies at a later age. Other audiological examinations, including immittance, auditory brainstem response, and distortion production otoacoustic emissions, revealed cochlear involvement. With the exception of one patient, all complained of highfrequency tinnitus around the onset of hearing loss, and none of them reported any vestibular symptoms. Comprehensive family medical histories and clinical examination showed no other clinical abnormalities. Computed tomography of the temporal bone in the proband was normal.

\section{Exome sequencing}

The exomes of three affected individuals (V: 5, VI: 4, and VI: 32) and one normal-hearing member (V: 4) from this family were sequenced to identify potential variants responsible for hearing loss. We generated an average of $5.9 \mathrm{~Gb}$ of sequence, with at least $80 \times$ average coverage for each individual as paired-end, 90-bp reads (Supplementary Figures S2 and S3 online). After mapping to the human reference, $\sim 98.55 \%$ of the targeted bases were covered sufficiently to pass quality assessment for calling SNPs and short indels (Supplementary Table S4 online). We identified an average of 17,397 SNPs (synonymous and nonsynonymous)

Table 1 Candidate variants shared by three affected individuals of family JSNY-028

\begin{tabular}{|c|c|c|c|c|c|c|}
\hline Chromosome & Position & Reference & Change & Gene & Codon & Substitution \\
\hline Chr2 & 131954220 & G & A & TUBA3D & GGC484AGC & G162S \\
\hline Chr4 & 100568026 & C & G & $A D H 7$ & GCT527GGT & A176G \\
\hline Chr4 & 109888769 & $-1 \mathrm{~A}$ & l & AGXT2L1 & Splice site & Deletion \\
\hline Chr9 & 40706296 & $A$ & C & FAM74A3 & ACC $448 C C C$ & T150P \\
\hline Chr9 & 124431591 & $+1 \mathrm{~A}$ & l & OR1B1 & Frameshift & Insertion \\
\hline Chr10 & $27463062-27463063$ & $-2 \mathrm{GA}$ & l & YME1L1 & Splice site & Deletion \\
\hline Chr12 & 131247613 & G & $\mathrm{T}$ & GALNT9 & CGG706TGG & R236W \\
\hline Chr20 & $60835152-60835153$ & $-2 \mathrm{CT}$ & I & OSBPL2 & Frameshift & Deletion \\
\hline Chr19 & 45075974 & A & G & $F C G B P$ & GTC9476GGC & V3159G \\
\hline Chr21 & 30642431 & G & C & KRTAP13-6P & GGC319CGC & G107R \\
\hline
\end{tabular}

The symbol "I" means not presenting the base substitution. 
in coding regions, an average of 2,270 variants (SNPs and indels) in introns that may affect splicing (within $10 \mathrm{bp}$ of the intron/ exon junction), and an average of 4,409 indels in coding regions or introns (Supplementary Table S5 online). Given that these patients are related and they are expected to share the causal variant for hearing loss, a total of 197 variants were screened after comparison with SNP databases (Supplementary Tables S6 and S7 online). Among them, 77 variants (including 66 nonsynonymous SNPs, splice acceptor and donor sites, and 11 indels) were predicted to potentially have a functional impact on the gene (Supplementary Tables S8 and S9 online). Via Sanger sequencing, we excluded false-positive results and identified 11 variants (including 7 rare SNPs and 4 indels) that cosegregated with the deafness phenotype in these three subjects (Table 1).

\section{Cosegregation analysis of OSBPL2 c.153_154deICT in JSNY- 028 family members}

We then performed cosegregation analysis by Sanger sequencing on the seven validated SNPs and four indels using the available 25 clinically affected subjects, 14 unaffected relatives, and 12 spouses of family JSNY-028 (Figure 1). As a result, a heterozygous 2-bp de novo deletion (c.153_154delCT) corresponding to codons 51/52 was detected in exon 3 of OSBPL2. This mutation caused a frameshift after the 52nd residue (serine) that would, if translated, produce 99 novel amino acids from codons 53 to 151 and terminate translation at codon 152 (p.Gln53Argfs $\left.{ }^{\star} 100\right)$ (codons 51/52 do not change for the same-sense mutations) (Figure 2a-c; Supplementary Figure S4 online). The results showed that 25 patients were heterozygous for this mutation, and none of the clinically unaffected family members and 300 ethnicity-matched controls carried this variant. Thus, the mutation of c.153_154delCT in OSBPL2 was completely cosegregated with the hearing loss phenotype of this family. To further evaluate the role of OSBPL2 in AD NSHL, we sequenced all of the exons and exon-intron boundaries of this gene in the available 25 affected members and did not find other mutations in the OSBPL2 gene. Except for the mutation of c.153_154delCT in the OSBPL2 gene, none of the candidate SNPs $(n=7)$ or indels $(n=3)$ were detected as being cosegregated with the hearing loss phenotype of family JSNY-028, as demonstrated by Sanger sequencing.

a \begin{tabular}{|l|l|l|l|l|l|l|l|l|l|l|l|l|l|l|l|l|l|l|l|l|l|l}
\hline $\mathrm{p} 13$ & $\mathrm{p} 12.3$ & $\mathrm{p} 12.2$ & $\mathrm{p} 12.1$ & $\mathrm{p} 11.23$ & $\mathrm{p} 11.21 .22$ & $\mathrm{q} 11.23$ & $\mathrm{q} 12$ & $\mathrm{q} 13.12$ & $\mathrm{q} 13.13$ & $\mathrm{q} 13.2$ & $\mathrm{q} 13.32$ & $\mathrm{q} 13.33$ \\
\hline
\end{tabular} 20q13.33: $60.813 .580-60.871 .268(57.69 \mathrm{~kb})$

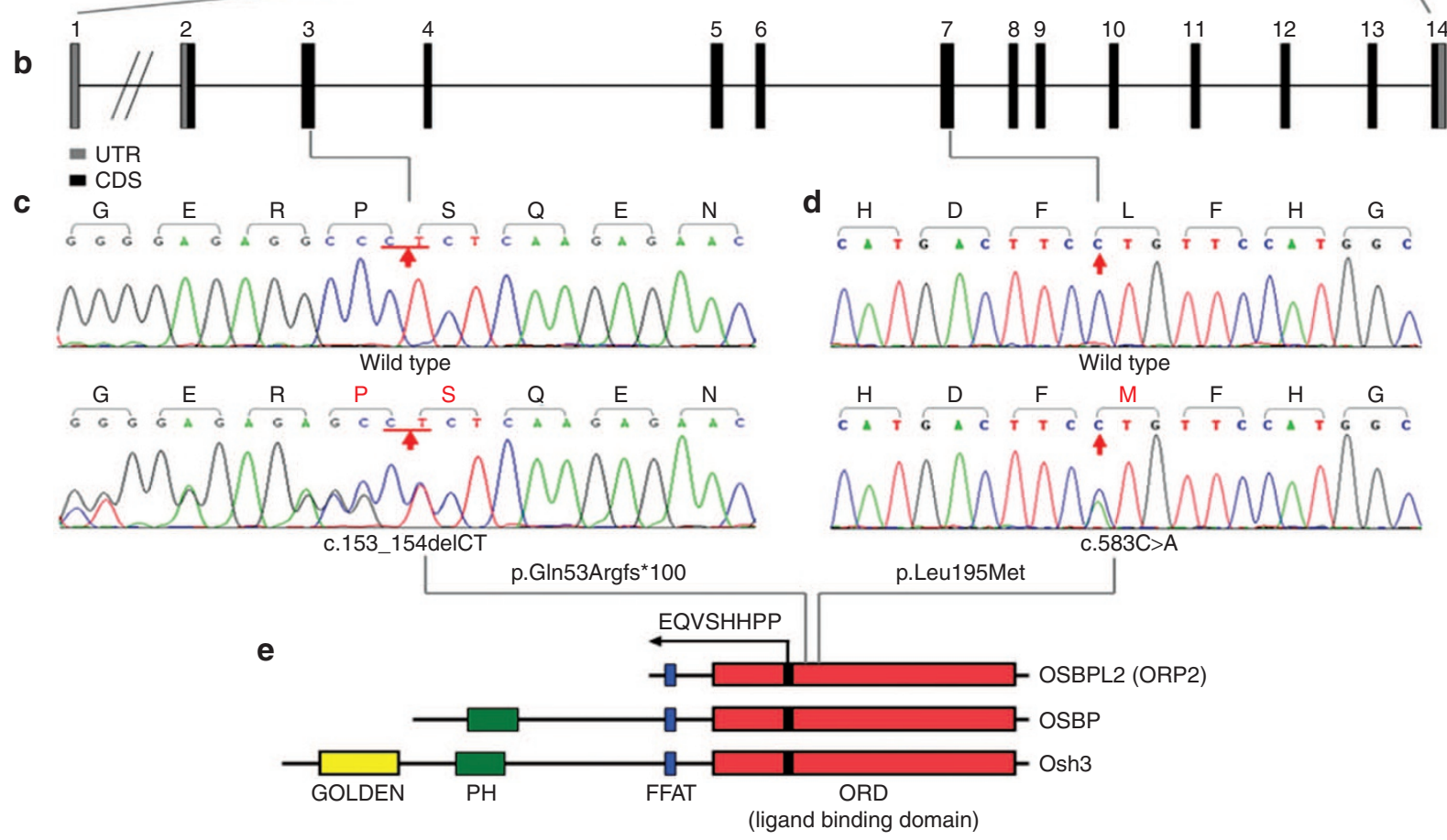

Figure 2 Schematic physical and genetic maps of the OSBPL2 gene (NC_000020.11, NM_144498.2, NP_653081.1, OMIM: 606731) and two heterozygous mutations found in the JSNY-028 family members and sporadic cases. (a) Schematic physical and genetic maps of OSBPL2, located in the 20q13.33 chromosomal region. (b) Structure of the OSBPL2 gene (14 exons). (c,d) Sequencing chromatograms of OSBPL2 showing two heterozygous mutations_c.153_154delCT (in exon 3, reverse sequencing) and c.583C >A (in exon 7, forward sequencing)—in affected individuals (bottom panel) as compared with those of normal controls (top panel). The mutated nucleotides are marked by red arrows. The predicted amino acid changes and surrounding amino acids are indicated above the sequences. (e) Alignment of OSBPL2, OSBP, and Osh3 protein sequences. OSBPL2 is composed of an FFAT motif (two phenylalanines in an acidic tract) and an OSBP-related domain with a highly conserved sequence motif, the "OSBP fingerprint," EQVSHHPP. OSBP, oxysterol binding protein; OSBPL2, oxysterol binding protein-like 2; Osh3, oxysterol binding protein homolog 3. 


\section{Screening of OSBPL2 mutations in sporadic subjects and AD NSHL pedigrees}

All exons and exon-intron boundaries of OSBPL2 in 452 sporadic NSHL cases and 38 probands of the unrelated AD NSHL pedigrees were sequenced. In one sporadic patient who had bilateral symmetric and moderate sensorineural hearing loss, we detected a missense variant c.583C $>$ A (p.Leu195Met) in exon 7 of OSBPL2 (Figure 2d), which was absent in 300 controls. In addition, the reported NSHL-causative mutations were not detected in this case. However, no family members were available for cosegregation analysis.

\section{Model building and structure-based analysis}

The full length of OSBPL2 complementary DNA encodes a deduced 480-amino acid residue protein with a predicted molecular mass of $55 \mathrm{kDa}$. The BLAST results indicated that OSBPL2 showed a significant homology to the ligandbinding domain of yeast oxysterol binding protein homolog 3 (Osh3) (holo-form) (Protein Data Bank ID: 4INQ; http:// blast.ncbi.nlm.nih.gov/) (Supplementary Figure S5 online). The PROCHECK program was used to check the detailed residue-by-residue stereochemical quality on the basis of a Ramachandran plot (Supplementary Figure S6 online), by which the modeled structure of OSBPL2 showed $69.2 \%$ of residues in the most favored regions, $29.2 \%$ of residues in additional and generously allowed regions, and only $1.6 \%$ of residues in the disallowed regions, which suggested that the modeled structures of OSBPL2 (Figure 3a) were logical and acceptable.

As shown in Figures 2 and 3, the c.153_154delCT (p.Gln53Argfs ${ }^{\star} 100$ ) and c.583C $>$ A (p.Leu195Met) mutations were located in the coding region of the OSBP-related domain (ORD) (residues 36-472), which was the most functional part of OSBPL2 for ligand binding. ${ }^{20-22}$ The mutation of c.153_154delCT led to a frameshift in coding sequences with a stop codon $\left(^{*}\right)$ at position 100 in the shifted reading frame, which resulted in changes of 99 amino acids (residuals 53-151) and in-frame deletion of 329 amino acids in the ORD region. Meanwhile, the modeling and structure-based analysis of OSBPL2 showed that the c.583C $>$ A (p.Leu195Met) mutation changes the shape of the protein in the ORD region (Figure $3 b$ ), which may alter its ability to bind ligands. Based on this information, it might be suggested that these two mutations could be functionally deleterious.

\section{Expression studies}

We evaluated the transcript expression of Osbpl2 in different adult mouse tissues using reverse transcription PCR. The Osbpl2 gene was strongly expressed in the mouse cochlea and brain, and it was also widely expressed in the heart, liver, spleen, lung, kidney, and muscle (Supplementary Figure S7 online). Immunoreactivity for Osbpl2 was localized to multiple structures in the specific area of the mouse cochlea. Intensive staining for Osbpl2 was detected in the stria vascularis, spiral ganglion, inner hair cells, and outer hair cells. Osbpl2 was also detected in the spiral ligament (Figure 4a). Higher-magnification views showed immunostaining for Osbpl2 in densely packed cytoplasmic structures of mostly positive cells (Figure $4 \mathrm{~b}$ ).

\section{DISCUSSION}

In this study a novel deafness-associated candidate gene, OSBPL2, was identified in a large Chinese family with AD NSHL using WES techniques. A heterozygous de novo

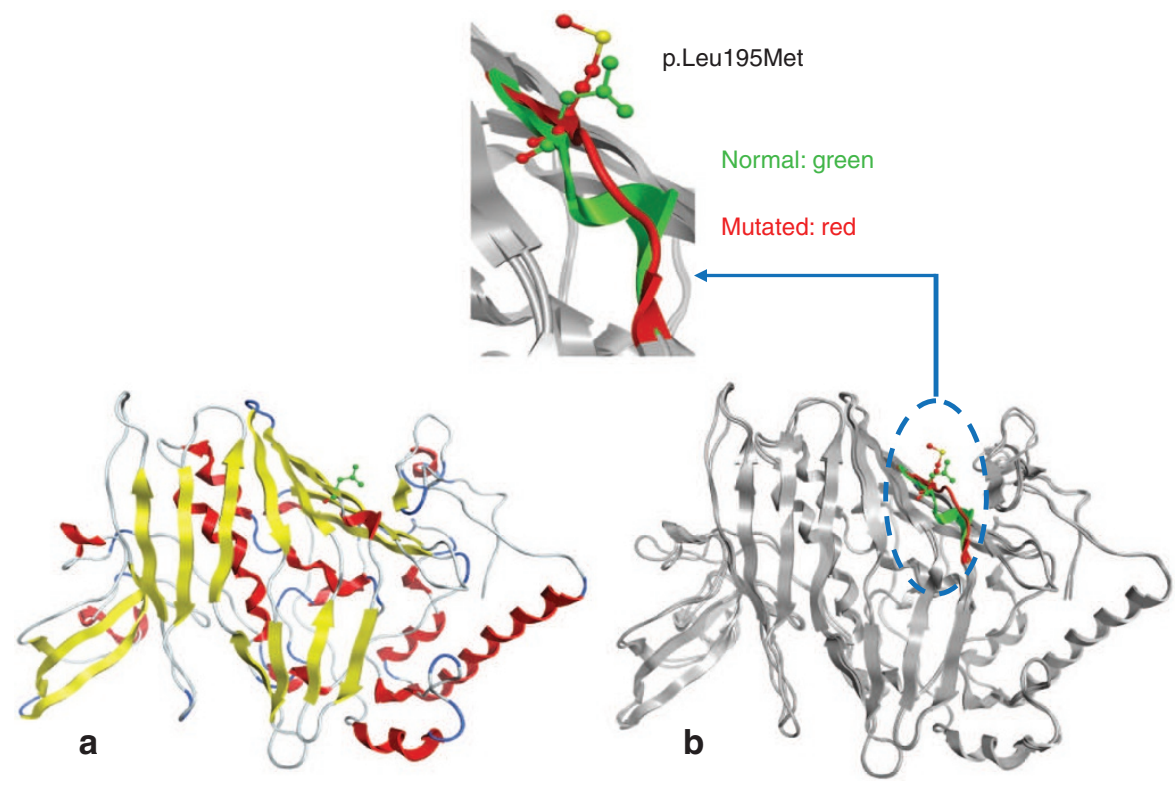

Figure 3 Comparison of normal and mutated OSBPL2 predicted structure. (a) Structure modeling of the normal protein. (b) Superimposed structure modeling of the normal and mutated protein; the mutated site of p.Leu195Met is emphasized by a blue circle and locally zoomed (normal in green with helix configuration; mutated in red with no helix configuration). OSBPL2, oxysterol binding protein-like 2. 

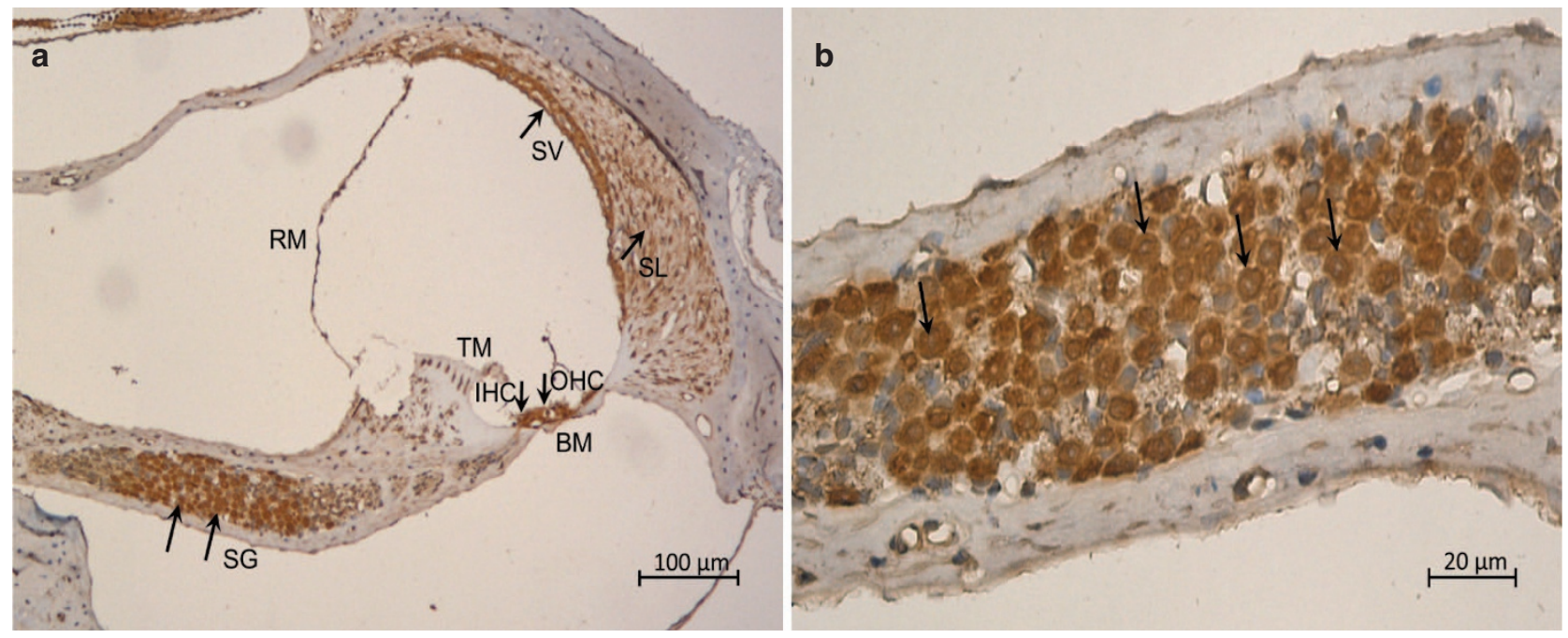

Figure 4 Immunohistochemical localization of Osbpl2 in a 12-week-old C57BL/6 mouse cochlea. (a) Low magnification of the mouse cochlea. Osbpl2 was strongly expressed in the stria vascularis (SV), spiral ganglion (SG), inner hair cells (IHCs), and outer hair cells (OHCs). Osbpl2 was also detected in the spiral ligament (SL). The scale bar represents $100 \mu \mathrm{m}$. (b) High magnification of the SG, showing intensive staining of some positive cells. The scale bar represents $20 \mu \mathrm{m}$. Osbpl2, oxysterol binding protein-like 2.

frameshift mutation (c.153_154delCT, p.Gln53Argfs*100) in exon 3 of OSBPL2 was identified as being completely cosegregated with the clinical phenotype of hearing loss in this family and absent in the controls. We speculate that the frameshift transcripts from the mutant allele in this family would most likely be caused by the induction of nonsense-mediated messenger RNA decay, resulting in a deficiency of the OSBPL2 protein. ${ }^{23,24}$ In addition, a missense mutation (c.583C $>A$ ) in exon 7 was detected in a sporadic case, which further supported the hypothesis that mutations of OSBPL2 might lead to pathogenicity and thereby the disease phenotype.

Oxysterol-binding protein (OSBP)-related proteins (ORPs), also known as OSBPLs, constitute a large and evolutionarily conserved family with numerous homologs identified in eukaryotic species from yeast to humans. ${ }^{25-28}$ Budding yeast has seven ORP genes (Osh1-Osh 7$)$, whereas the mammalian OSBP/ORP family consists of a large number of proteins encoded by 12 genes. ${ }^{27}$ Since OSBP was first identified in the mid-1980s by Taylor et al. ${ }^{29}$ as a soluble, high-affinity receptor, evidence has accumulated indicating that the OSBP/ORP family plays important roles in regulating cellular lipid homeostasis and in many cellular processes, including cell signaling, vesicular trafficking, lipid metabolism, and particularly sterol signaling or sterol transport functions. ${ }^{30-32}$

OSBPL2 (ORP2) is a member of the OSBP/ORP family and is also the only mammalian isoform that is expressed exclusively as a truncated short ORP lacking the pleckstrin homology and Golgi dynamics domains, which are found in other OSBP/ORP family members, but harboring a FFAT (two phenylalanines in an acidic tract) motif and an ORD region (Figure 2e). It is known that a common feature of all ORPs is the conserved $C$-terminal ORD. ${ }^{30,31}$ Tong et al. ${ }^{32}$ proved that the unifying role in all ORP homologs was the binding of PI[4]P to ORD, with additional sterol binding for certain homologs, and yeast complementation tests showed that PI[4]P binding to ORD is essential for function. A strictly conserved OSBP-fingerprint motif, "EQVSHHPP," in the ORD region of OSBPL2 is a specific binding motif for the head of the group of the PI[4]P ligand, suggesting roles as phosphoinositide-binding proteins ${ }^{20-22}$ (Figure 2e). Although OSBPL2 does not have a pleckstrin homology domain, it had been reported that the ORD region of OSBPL2 plays a role in binding anionic phospholipids analogous to yeast Osh 3 and Osh4p. ${ }^{32,33} \mathrm{PI}$ [4]P binding activity could be responsible for targeting of OSBPL2 to the Golgi apparatus, where it disrupted endoplasmic reticulum-Golgi trafficking when overexpressed in Chinese hamster ovary cells. ${ }^{20,34}$ It has been found that OSBPL2 was localized to the surface of lipid droplets, which are sites of storage for neutral lipid triglycerides and cholesterol esters, and that it dissociated to the cytoplasm upon the addition of exogenous 22(R)-hydroxycholesterol. Knockdown of OSBPL2 expression with RNA interference increased the amounts of triglyceride and cholesterol ester. ${ }^{22}$ The presence of OSBPL2 on lipid droplets was consistent with an inhibitory role in triglyceride, phospholipid, and cholesterol ester metabolism. ${ }^{20,21}$ This evidence suggests that OSBPL2 plays a major role in the integrated control of cholesterol and triglyceride metabolism. In fact, the role of lipids in cochlear physiology and pathology is yet unexplored, but lipids, especially cholesterol and triglyceride concentrations in some diseases such as diabetes and hyperlipidemia, are receiving more attention now because of clinical studies showing that several modifiable factors might affect hearing. However, the molecular mechanisms of lipid abnormalities associated with hearing function still remain elusive, a state that may persist for a long time. ${ }^{35,36}$ If the presented OSBPL2, lipid metabolism, and hearing are linked, a new area of possible intervention for the genetic study of hearing function in these special populations will be opened. 
Indeed, the challenge before us is determining how to link OSBPL2 or OSBP/ORPs with hearing impairment in this study. Although several members of the OSBP/ORP family have been putatively associated with some diseases, ${ }^{37-39}$ the implication in hearing impairment had not been previously reported, and little was known about the relationship between OSBP/ORPs (including other lipid transport proteins) and the cell physiology of the inner ear. Laitinen et al. ${ }^{20}$ proved that OSBPL2 was expressed in almost all human tissues, but the inner ear or cochlea was not included in that work, and almost nothing was known about the role of OSBPL2 in the human ear. In this study, we confirmed the expression of the Osbpl2 gene in the mouse cochlea as well as in other mouse tissues via reverse transcription PCR and immunohistochemistry methods. In addition, Adams showed that strong expression of the Osbpl2 gene was detected in the cochlea of 9-week-old mouse using a microarray assay (http://research.meei.harvard.edu/ Otopathology/tbimages/mouse.html). Based on the data mentioned above, it was suggested that OSBPL2 expression in the cochlea might have a functional impact on hearing regulation. Considering the function of OSBPL2 and the role of the ORD region mentioned above, as well as structure-based analysis via modeling of normal and mutated protein structures, it was speculated that the c.153_154delCT and c.583C $>$ A mutations in OSBPL2, located in the coding sequence of the ORD region, could have a functionally deleterious impact on the ability of OSBPL2 to bind ligands and cell receptors, which might be the causative factor of disorders with a deafness phenotype. Among the reported NSHL-causative loci, DFNB 65 was the only locus located on chromosome 20 (20q13.2-q13.32). ${ }^{40}$ Interestingly, OSBPL2 was also located on chromosome 20 (20q13.33), just adjacent to DFNB65, and this is the first time that a candidate $\mathrm{AD}$ NSHL-causative gene has been identified on human chromosome 20.

In summary, via the WES approach, as well as structural modeling and functional prediction, we identified OSBPL2 as a novel candidate gene for progressive AD NSHL. However, there were no data comparing the expression of OSBP/ORPs in human cochlea and other tissues, and no functional assessments of OSBPL2 variants producing the deafness phenotype were previously reported. Further functional studies of this specific gene are still needed to gain insights into the pathogenic mechanisms involved in NSHL.

\section{SUPPLEMENTARY MATERIAL}

Supplementary material is linked to the online version of the paper at http://www.nature.com/gim

\section{ACKNOWLEDGMENTS}

This research was supported by a grant from the National Natural Science Foundation of China (31171217) to X.C. and grants from Jiangsu Health Administration of China (LJ201120) and the Research Special Fund for Public Welfare Industry of Health, Ministry of Health of China (201202005) to G.X.

\section{DISCLOSURE}

The authors declare no conflict of interest.

\section{REFERENCES}

1. Cohen MM, Gorlin RJ. Hereditary hearing loss and its syndromes. In: Gorlin RJ, Toriello HV, Cohen MM (eds). Oxford Monographs on Medical Genetics, No 28 Oxford University Press: New York, 1995:9-21.

2. Keats BJB, Berlin Cl, Gregory P. Epidemiology of genetic hearing loss. Semin Hear 2006; 7:136-147.

3. Dror AA, Avraham KB. Hearing loss: mechanisms revealed by genetics and cell biology. Annu Rev Genet 2009;43:411-437.

4. Jones S, Hruban RH, Kamiyama M, et al. Exomic sequencing identifies PALB2 as a pancreatic cancer susceptibility gene. Science 2009;324:217.

5. Ng SB, Turner EH, Robertson PD, et al. Targeted capture and massively parallel sequencing of 12 human exomes. Nature 2009;461:272-276.

6. $\mathrm{Ng} \mathrm{SB}$, Buckingham KJ, Lee $\mathrm{C}$, et al. Exome sequencing identifies the cause of a mendelian disorder. Nat Genet 2010;42:30-35.

7. Bilgüvar K, Oztürk AK, Louvi A, et al. Whole-exome sequencing identifies recessive WDR62 mutations in severe brain malformations. Nature 2010;467:207-210.

8. Wang JL, Yang X, Xia K, et al. TGM6 identified as a novel causative gene of spinocerebellar ataxias using exome sequencing. Brain 2010;133(Pt 12):3510 3518.

9. Liu Y, Gao M, Lv YM, et al. Confirmation by exome sequencing of the pathogenic role of NCSTN mutations in acne inversa (hidradenitis suppurativa). J Invest Dermato/ 2011;131:1570-1572.

10. Coppieters F, Van Schil K, Bauwens M, et al. Identity-by-descent-guided mutation analysis and exome sequencing in consanguineous families reveals unusual clinical and molecular findings in retinal dystrophy. Genet Med 2014;16:669-678.

11. Boycott KM, Dyment DA, Sawyer SL, Vanstone MR, Beaulieu CL. Identification of genes for childhood heritable diseases. Annu Rev Med 2014;65:19-31.

12. Walsh $T$, Shahin H, Elkan-Miller $T$, et al. Whole exome sequencing and homozygosity mapping identify mutation in the cell polarity protein GPSM2 as the cause of nonsyndromic hearing loss DFNB82. Am J Hum Genet 2010;87:90-94

13. Klein CJ, Botuyan MV, Wu Y, et al. Mutations in DNMT1 cause hereditary sensory neuropathy with dementia and hearing loss. Nat Genet 2011;43:595600.

14. Girotto G, Abdulhadi K, Buniello A, et al. Linkage study and exome sequencing identify a BDP1 mutation associated with hereditary hearing loss. PLOS One 2013;8:e80323.

15. Jaworek TJ, Richard EM, Ivanova AA, et al. An alteration in ELMOD3, an Arl2 GTPase-activating protein, is associated with hearing impairment in humans. PLoS Genet 2013;9:e1003774.

16. Zhao $Y$, Zhao F, Zong $L$, et al. Exome sequencing and linkage analysis identified tenascin-C (TNC) as a novel causative gene in nonsyndromic hearing loss. PLOS One 2013;8:e69549.

17. Imtiaz A, Kohrman DC, Naz S. A frameshift mutation in GRXCR2 causes recessively inherited hearing loss. Hum Mutat 2014;35:618-624.

18. Santos-Cortez RL, Lee K, Giese AP, et al.; University of Washington Center for Mendelian Genomics. Adenylate cyclase 1 (ADCY1) mutations cause recessive hearing impairment in humans and defects in hair cell function and hearing in zebrafish. Hum Mol Genet 2014;23:3289-3298.

19. Majewski J, Rosenblatt DS. Exome and whole-genome sequencing for gene discovery: the future is now! Hum Mutat 2012;33:591-592.

20. Laitinen S, Lehto M, Lehtonen S, et al. ORP2, a homolog of oxysterol binding protein, regulates cellular cholesterol metabolism. J Lipid Res 2002;43:245255.

21. Käkelä R, Tanhuanpää K, Laitinen S, Somerharju P, Olkkonen VM. Overexpression of OSBP-related protein 2 (ORP2) in CHO cells induces alterations of phospholipid species composition. Biochem Cell Biol 2005;83:677-683.

22. Hynynen R, Suchanek M, Spandl J, Bäck N, Thiele C, Olkkonen VM. OSBPrelated protein 2 is a sterol receptor on lipid droplets that regulates the metabolism of neutral lipids. J Lipid Res 2009;50:1305-1315.

23. Nagy E, Maquat LE. A rule for termination-codon position within introncontaining genes: when nonsense affects RNA abundance. Trends Biochem Sci 1998;23:198-199. 
24. Kervestin $S$, Jacobson A. NMD: a multifaceted response to premature translational termination. Nat Rev Mol Cell Biol 2012;13:700-712.

25. Beh CT, Cool L, Phillips J, Rine J. Overlapping functions of the yeast oxysterolbinding protein homologues. Genetics 2001;157:1117-1140.

26. Lehto $M$, Laitinen $S$, Chinetti $G$, et al. The OSBP-related protein family in humans. J Lipid Res 2001;42:1203-1213.

27. Jaworski CJ, Moreira E, Li A, Lee R, Rodriguez IR. A family of 12 human genes containing oxysterol-binding domains. Genomics 2001;78: 185-196.

28. Zeng B, Zhu G. Two distinct oxysterol binding protein-related proteins in the parasitic protist Cryptosporidium parvum (Apicomplexa). Biochem Biophys Res Commun 2006;346:591-599.

29. Taylor FR, Saucier SE, Shown EP, Parish EJ, Kandutsch AA. Correlation between oxysterol binding to a cytosolic binding protein and potency in the repression of hydroxymethylglutaryl coenzyme A reductase. J Biol Chem 1984;259:1238212387.

30. Raychaudhuri S, Prinz WA. The diverse functions of oxysterol-binding proteins. Annu Rev Cell Dev Biol 2010;26:157-177.

31. Beh CT, McMaster CR, Kozminski KG, Menon AK. A detour for yeast oxysterol binding proteins. J Biol Chem 2012;287:11481-11488.

32. Tong J, Yang H, Yang H, Eom SH, Im YJ. Structure of Osh3 reveals a conserved mode of phosphoinositide binding in oxysterol-binding proteins. Structure 2013;21:1203-1213.
33. Li X, Rivas MP, Fang M, et al. Analysis of oxysterol binding protein homologue Kes $1 p$ function in regulation of Sec14p-dependent protein transport from the yeast Golgi complex. J Cell Biol 2002;157:63-77.

34. Xu Y, Liu Y, Ridgway ND, McMaster CR. Novel members of the human oxysterolbinding protein family bind phospholipids and regulate vesicle transport. J Biol Chem 2001;276:18407-18414.

35. Weng T, Devine EE, Xu H, Yan Z, Dong P. A clinical study of serum lipid disturbance in Chinese patients with sudden deafness. Lipids Health Dis 2013:12:95.

36. Simpson AN, Matthews LJ, Dubno JR. Lipid and C-reactive protein levels as risk factors for hearing loss in older adults. Otolaryngol Head Neck Surg 2013;148:664-670.

37. Wang PY, Weng J, Anderson RG. OSBP is a cholesterol-regulated scaffolding protein in control of ERK 1/2 activation. Science 2005;307:1472-1476.

38. Yan $D$, Lehto $M$, Rasilainen $L$, et al. Oxysterol binding protein induces upregulation of SREBP-1C and enhances hepatic lipogenesis. Arterioscler Thromb Vasc Biol 2007:27:1108-1114.

39. Bouchard L, Faucher G, Tchernof A, et al. Association of OSBPL11 gene polymorphisms with cardiovascular disease risk factors in obesity. Obesity (Silver Spring) 2009;17:1466-1472.

40. Tariq A, Santos RL, Khan MN, et al. Localization of a novel autosomal recessive nonsyndromic hearing impairment locus DFNB65 to chromosome 20q13.2-q13.32. J Mol Med (Berl) 2006;84:484-490. 